University of Nebraska - Lincoln

DigitalCommons@University of Nebraska - Lincoln

\title{
Expanded 3D Nanofiber Scaffolds: Cell Penetration, Neovascularization, and Host Response
}

\author{
Jiang Jiang \\ University of Nebraska Medical Center, jiang.jiang@unmc.edu \\ Zhuoran Li \\ University of Nebraska-Lincoln \\ Hongjun Wang \\ University of Nebraska Medical Center, hongjun.wang@unmc.edu \\ Yue Wang \\ University of Nebraska Medical Center \\ Mark A. Carlson \\ University of Nebraska Medical Center, Omaha, macarlso@unmc.edu
}

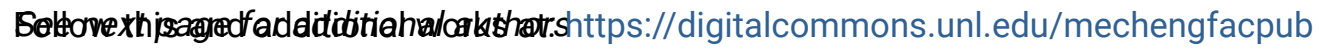

Part of the Biomechanics and Biotransport Commons, Biomedical Devices and Instrumentation Commons, Mechanics of Materials Commons, Nanoscience and Nanotechnology Commons, Other Engineering Science and Materials Commons, and the Other Mechanical Engineering Commons

Jiang, Jiang; Li, Zhuoran; Wang, Hongjun; Wang, Yue; Carlson, Mark A.; Teusink, Matthew J.; MacEwan, Matthew R.; Gu, Linxia; and Xie, Jingwei, "Expanded 3D Nanofiber Scaffolds: Cell Penetration, Neovascularization, and Host Response" (2016). Mechanical \& Materials Engineering Faculty Publications. 170.

https://digitalcommons.unl.edu/mechengfacpub/170

This Article is brought to you for free and open access by the Mechanical \& Materials Engineering, Department of at DigitalCommons@University of Nebraska - Lincoln. It has been accepted for inclusion in Mechanical \& Materials Engineering Faculty Publications by an authorized administrator of DigitalCommons@University of Nebraska Lincoln. 


\section{Authors}

Jiang Jiang, Zhuoran Li, Hongjun Wang, Yue Wang, Mark A. Carlson, Matthew J. Teusink, Matthew R. MacEwan, Linxia Gu, and Jingwei Xie 


\title{
Expanded 3D Nanofiber Scaffolds: Cell Penetration, Neovascularization, and Host Response
}

\author{
Jiang Jiang, Zhuoran Li, Hongjun Wang, Yue Wang, Mark A. Carlson, \\ Matthew J. Teusink, Matthew R. MacEwan, Linxia Gu, and Jingwei Xie*
}

Herein, a robust method to fabricate expanded nanofiber scaffolds with controlled size and thickness using a customized mold during the modified gas-foaming process is reported. The expansion of nanofiber membranes is also simulated using a computational fluid model. Expanded nanofiber scaffolds implanted subcutaneously in rats show cellular infiltration, whereas non-expanded scaffolds only have surface cellular attachment. Compared to unexpanded nanofiber scaffolds, more $\mathrm{CD}^{+} 8^{+}$and $\mathrm{CD} 163^{+}$cells are observed within expanded scaffolds at all tested time points post-implantation. More $\mathrm{CCR7}^{+}$cells appear within expanded scaffolds at week 8 post-implantation. In addition, new blood vessels are present within the expanded scaffolds at week 2. The formed multinucleated giant cells within expanded scaffolds are heterogeneous expressing CD68, CCR7, or CD163 markers. Together, the present study demonstrates that the expanded nanofiber scaffolds promote cellular infiltration/tissue integration, a regenerative response, and neovascularization after subcutaneous implantation in rats. The use of expanded electrospun nanofiber scaffolds offers a promising method for in situ tissue repair/regeneration and generation of 3D tissue models/constructs.

\section{Introduction}

Electrospinning is an enabling technology that can produce a matrix reminiscent of extracellular matrix found in a variety of tissues and organs, which are synthesized and hierarchically organized into fibrillar form with fiber dimensions down to nanometer scale. ${ }^{[1-3]}$ Electrospun nanofibers can also provide a large surface area-to-volume ratio ideal for immobilizing bioactive signaling molecules and pharmaceutical agents capable of positively influencing cellular behavior. ${ }^{[4]}$ Nanotopographic cues rendered by electrospun nanofibers have demonstrated the ability to guide cellular morphology, migration, and differentiation. ${ }^{[5]}$ However, one major drawback that lies in traditional electrospun nanofiber membranes is the small pore size resulting from tightly packed nanofiber layers, which limits cellular infiltration and 3D tissue formation. ${ }^{[6]}$ 3D scaffolds composed of electrospun nanofibers that promote cellular infiltration therefore may represent an improved synthetic matrix for use in tissue repair/ regeneration, and provide a better mimic for both the structure and composition of targeted tissues. ${ }^{[7]}$

In order to improve the modeling of native tissue structure, many attempts have been made to fabricate 3D electrospun nanofiber scaffolds. Jun and co-workers designed a unique collector consisting of a grounded spherical dish and an array of needle-like probes for creation of a focused, low density, uncompressed poly $(\varepsilon$ caprolactone) (PCL) nanofiber scaffold. ${ }^{[8]}$ In order to promote electron transfer from deposited fibers to the collector and achieve repulsion between deposited fibers and the collector during electrospinning, Yang and co-workers used a negatively charged spinneret and a positively charged collector
Dr. J. Jiang, H. Wang, Y. Wang, Prof. J. Xie

Department of Surgery-Transplant and Mary \& Dick Holland

Regenerative Medicine Program

University of Nebraska Medical Center

Omaha, NE 68198, USA

E-mail: jingwei.xie@unmc.edu

Z. Li, Prof. L. Gu

Department of Mechanical and Materials Engineering

University of Nebraska-Lincoln

Lincoln, NE 68588, USA

Prof. M. A. Carlson

Department of Surgery and Department of Genetics

Cell Biology and Anatomy

University of Nebraska Medical Center

Omaha, NE 68198, USA
Prof. M. A. Carlson

Department of Surgery

VA Nebraska-Western lowa Health Care System

Omaha, NE 68105, USA

Prof. M. J. Teusink

Department of Orthopedic Surgery and Rehabilitation

University of Nebraska Medical Center

Omaha, NE 68198, USA

Prof. M. R. MacEwan

Department of Neurosurgery

Washington University School of Medicine

Saint Louis, MO 63110, USA

DOI: 10.1002/adhm.201600808 
board, electrospinning zein, and keratin scaffolds with fibers oriented randomly and evenly in $3 \mathrm{D} . .^{[9]}$ Based on a similar principle, Jang and co-workers added salts to a polymer solution prior to electrospinning and produced a sponge nanofiber matrix composed of multilayered nanofiber sheets. ${ }^{[10]}$ Kwon and co-workers exposed 2D poly(L-lactide) (PLLA) nanofiber membranes to ultrasonication with different times and obtained 3D nanofiber scaffolds with adjustable pore size and thickness with longer treatment. ${ }^{[11]}$ Alsberg and co-workers further combined fiber-fiber charge repulsions and ultrasonication to fabricate highly porous alginate nanofiber scaffolds. ${ }^{[12]}$ Unfortunately, most studies have been limited to the fabrication of 3D nanofiber scaffolds composed of random nanofibers and/or certain materials. The resultant scaffolds often had insufficient thickness, restricted geometries, and/ or uncontrolled porosity. ${ }^{[8-12]}$ These fabricated scaffolds were also associated with unordered structures and lack of nanotopographic cues that are critical for regeneration of highly organized tissues such as tendon, nerve, and muscle.[13] In addition, most of these 3D nanofiber scaffolds were only examined either in vitro or in vivo with respect to cellular infiltration, lacking the characterization of host response and neovascularization.

Our recent study reported a modified gas-foaming approach to expand electrospun nanofiber membranes in the third dimension. ${ }^{[13]}$ The expanded nanofiber scaffolds had significantly higher porosity than traditional 2D nanofiber membranes, while maintaining aligned nanotopography. The expanded scaffolds also had a layered structure with gap widths and layer thicknesses controllable on micrometer scale, ideal for cell seeding and penetration. Robust cellular infiltration and proliferation within expanded nanofiber scaffolds were demonstrated in vitro. However, the usable size and precise control of scaffold thickness were not fully realized in previous work. ${ }^{[13]}$ For example, the largest size of fiber samples tested for expansion was $\approx 1 \mathrm{~cm} \times 1 \mathrm{~cm} .{ }^{[13]}$ Larger sizes of nanofiber membranes were not examined. Furthermore, the thickness of scaffolds fabricated was primarily dependent on processing time. In order to optimize this process for use in the production of scaffolds for tissue repair/regeneration, a greater range of scaffold sizes (width, length) and thicknesses are required. More precise control of scaffold thickness is also desired in order to optimize the translational potential of fabricated 3D nanofiber matrices. Therefore, a robust method capable of producing expanded electrospun nanofiber scaffolds with increased size and precise control of thickness is greatly needed. In addition, the previous study only tested the in vitro cellular infiltration for expanded nanofiber scaffolds. ${ }^{[13]}$ The in vivo response of expanded nanofiber scaffolds largely remains unknown. The objectives of the present study were to fabricate expanded nanofiber scaffolds with increased size and precise control of thickness, and to examine the cellular infiltration, host response, and neovascularization of expanded scaffolds after subcutaneous implantation in rats. The findings of this study aim to provide insight into the design of $3 \mathrm{D}$ biomimetic scaffolds for tissue repair and regeneration in situ and engineering 3D tissue models/constructs in vitro.

\section{Results}

\subsection{Fabrication and Characterization of Expanded Nanofiber Scaffolds}

Previously we demonstrated that electrospun nanofiber mats can be expanded in the third dimension after treatment with aqueous $\mathrm{NaBH}_{4}$ solution. ${ }^{[13]}$ However, the working size and thickness control of these expanded scaffolds were somewhat limited. In the present report, we have described a robust approach for fabrication of expanded nanofiber scaffolds with both a greater mat size and a precise control of thickness by using a customized glass mold (Figure 1). We were able to expand nanofiber scaffolds with a starting mat size of $80 \mathrm{~mm} \times 80 \mathrm{~mm}$ (increased from $10 \mathrm{~mm} \times 10 \mathrm{~mm}$; see Figure S1, Supporting Information). In addition, the precise thickness control of these expanded scaffolds was achieved using pre-designed glass spacers (Figure 1a).

Figure 2a shows the photographs of an unexpanded nanofiber mat (left) and freeze-dried, expanded nanofiber scaffolds $(30 \mathrm{~mm} \times 50 \mathrm{~mm}$ ) with thicknesses of $3 \mathrm{~mm}$ (middle) and $10 \mathrm{~mm}$ (left). Figure $2 \mathrm{~b}, \mathrm{c}$ shows the photographs of the sterilized 3 and $10 \mathrm{~mm}$ thick scaffolds immersed in saline before subcutaneous implantation. Figure $2 \mathrm{~d}$ shows scanning electron microscopy (SEM) images of the cross section of an unexpanded PCL nanofiber mat, indicating that the fiber mat prior to expansion was composed of densely packed random nanofibers. Figure 2e,f shows SEM images of the cross sections of expanded 3 and $10 \mathrm{~mm}$ thick nanofiber scaffolds, displaying layered structures. Increased gap distances were observed for thicker nanofiber scaffolds. However, it seems that there was not much difference between the layer thicknesses of 3 and $10 \mathrm{~mm}$ thick nanofiber scaffolds. Based on SEM images, we also quantified the distribution of gap distances and layer thicknesses for the cross sections of 3 and $10 \mathrm{~mm}$ thick nanofiber scaffolds. Figure $2 \mathrm{~g}$ shows the distributions of gap distances for the cross sections. Thicker scaffolds had larger gap distances as the peak shifted to the right with increasing the thickness. The gap distances corresponding to the peaks were 10 and $20 \mu \mathrm{m}$ on the cross sections of 3 and $10 \mathrm{~mm}$ thick nanofiber scaffolds. Interestingly, the distributions of layer thicknesses were similar (Figure $2 \mathrm{~h}$ ). The layer thickness corresponding to the peak was around $10 \mu \mathrm{m}$.

\subsection{Simulation of Expanding Process of Nanofiber Membranes}

The volume fractions of the gas during the expanding process, including the bubble nucleation, growth, and coalescence, were described in Figure 3. The volume of fluid (VOF) model tracked the volumes of gas in each numerical grid. Specifically, the blue region corresponds to near zero volume fraction of gas, indicating a liquid phase. The red region with a unity volume fraction indicated a gas phase. Here we tracked the gas-liquid interface based on a given volume fraction of 0.5 , i.e., each grid at the interface that is half full with gas. Five inlets were constructed at the bottom layer to initiate the gas flow and mimic the gas bubble nucleation (Figure S2, Supporting Information). 

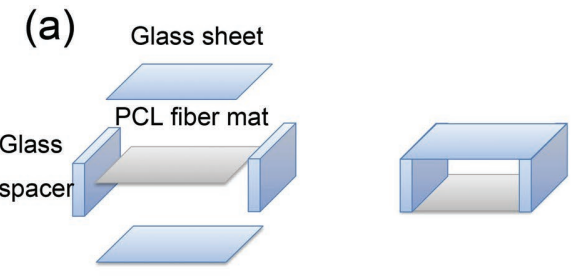

\section{Mold Assembly}

(d)

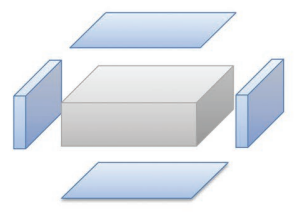

Remove mold

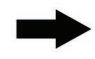

(b)

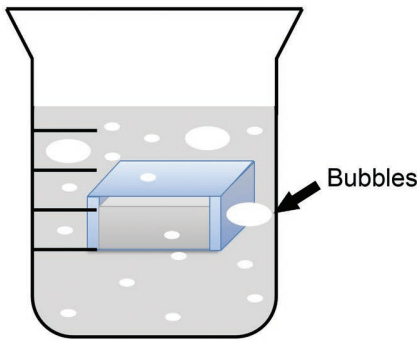

Expand \& Rinse $\downarrow$

(c)

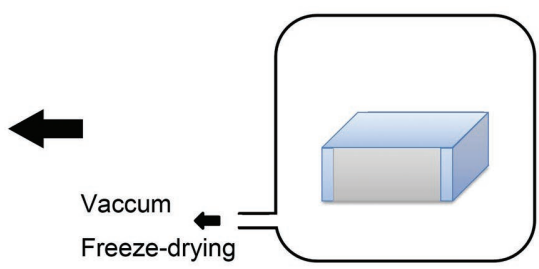

Freeze-drying

Figure 1. Schematic illustration of the fabrication of expanded PCL nanofiber scaffolds. a) A customized mold was made by a pair of glass sheets and spacers, which was assembled by glue. A piece of plasma-treated PCL fiber mat was placed at the center of the mold. b) The PCL fiber mat together with the mold was expanded in $1 \mathrm{M} \mathrm{NaBH}_{4}$ solution for $1 \mathrm{~h}$ at room temperature. c) The expanded PCL nanofiber scaffold was rinsed, vacuumed, and freeze-dried using a lyophilizer. d) The glass mold was gently removed and the expanded PCL nanofiber scaffold was ready to be sterilized and used for implantation.

It is clear that the nucleated bubbles grow bigger with the continued production of gas till coalescence of adjacent bubbles starting at $0.0003 \mathrm{~s}$. A short distance between two nucleation sites led to earlier gas bubble coalescence. At $0.0011 \mathrm{~s}$, a bubble layer was formed to further expand the nanofiber scaffolds. This process was also speculated in our previous work. ${ }^{[13]}$ Results also demonstrated that gas bubbles bridging two layers of scaffold were noncircular, which was different from classical free-floating gas bubbles. This could be due to the bubble surface tension and the pressure gradient at both ends of the scaffold. The flow dynamics of the expanding process could be further used to optimize and control the spatial structure of nanofiber scaffolds.

\subsection{Qualitative Histology Analysis}

In order to examine the cellular infiltration after subcutaneous implantation, we evaluated histological sections of the implanted PCL scaffolds and surrounding tissues with hematoxylin and eosin (H\&E) and Masson trichrome staining. Figure 4 shows H\&E staining of various scaffolds with surrounding tissues after subcutaneous implantation for 1, 2, 4, and 8 weeks. Unexpanded scaffolds only exhibited limited cellular penetration (Figure 4), except at occasional cracks within the scaffolds. In the expanded $3 \mathrm{~mm}$ thick scaffolds, cellular penetration was present from week 1 ; near complete penetration was present by week 8 . For the expanded $10 \mathrm{~mm}$ thick scaffolds, complete cellular penetration was present at week 8 . We also observed new blood vessel formation within expanded scaffolds at week 2, 4, and 8 , as indicated by the red blood cells (insets in Figure 4). In contrast, no blood vessel formation was noted within the unexpanded scaffolds.

Figure 5 shows Masson's trichrome staining of scaffolds with surrounding tissue after explantation. The cellular infiltration and new blood vessel formation showed a trend similar to the H\&E staining (Figure 4). In addition, initial collagen encapsulation of the implants was evident one week after implantation. Qualitatively, the thickness of the collagen capsule on unexpanded scaffolds tended to increase with time; this progression was not apparent on the expanded scaffolds. In addition, within-scaffold collagen deposition by infiltrated cells was observed within the expanded nanofiber scaffolds at week 4 and 8 (Figure 5). Multinucleated giant cells were present at the edge of unexpanded scaffolds and $3 \mathrm{~mm}$ thick expanded scaffolds at week 1, but not in the $10 \mathrm{~mm}$ expanded scaffolds (Figure 6). The giant cells in unexpanded scaffolds mostly were observed on the scaffold edges, while giant cells in expanded random scaffolds mostly were adjacent to the newly formed blood vessels. Similar results were observed for expanded aligned PCL scaffolds (Figure S3, Supporting Information).

\subsection{Quantification of Cell Infiltration, Neovascularization, and Host Response}

In order to fully understand the cellular infiltration, neovascularization, and host response, we quantified the depth of cell penetration into scaffolds, blood vessel density within scaffolds, the thickness of collagen fibrous capsules, and the number of 

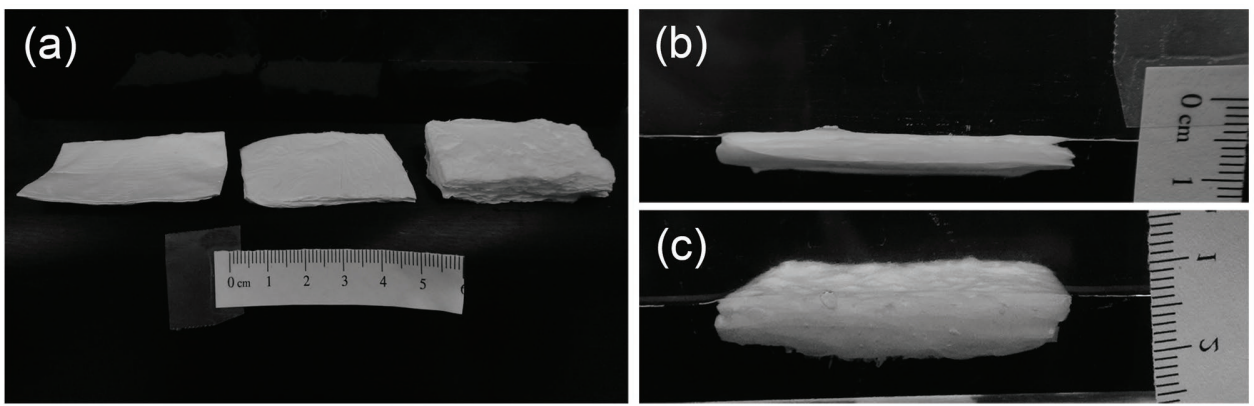

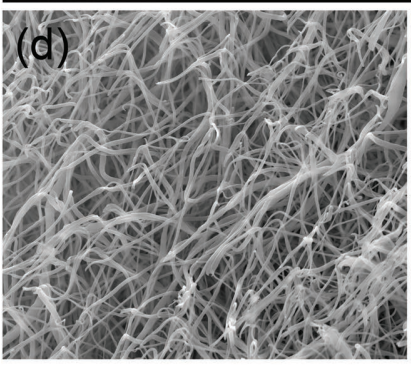

(g)

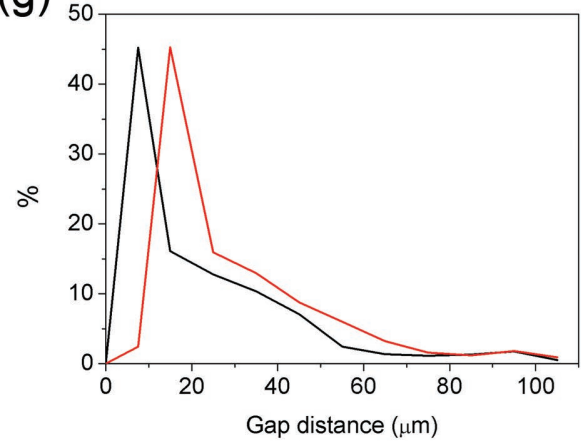

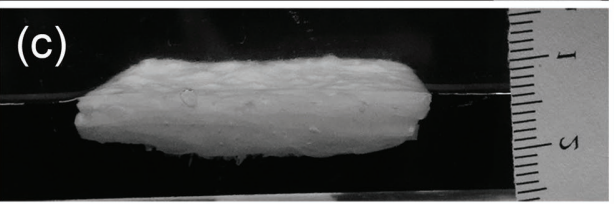
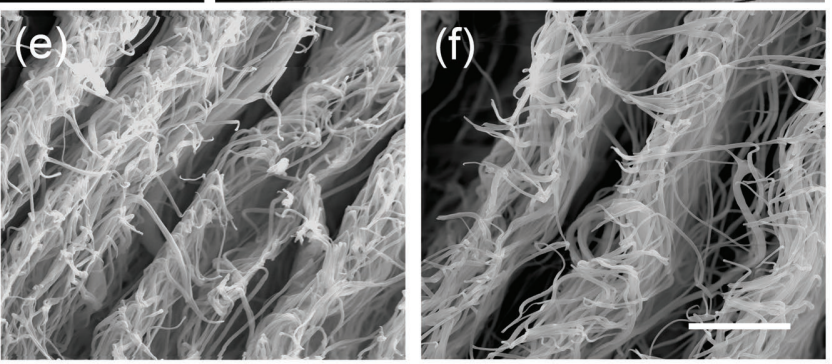

(h)

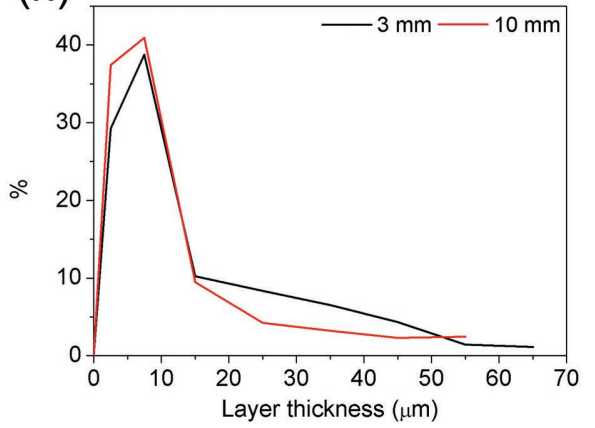

Figure 2. Morphological and structural characterizations of unexpanded and expanded random nanofiber scaffolds. a) Photographs of random PCL nanofiber scaffolds prior to and after treatment with $1 \mathrm{~m} \mathrm{NaBH}$ for $1 \mathrm{~h}$ using 3 and $10 \mathrm{~mm}$ molds. b,c) Photographs of expanded 3 and $10 \mathrm{~mm}$ thick scaffolds in saline. SEM images showing the cross sections of random PCL fiber mats d) before and after expansion with e) 3 and f) 10 mm molds. The scale bar in (d) $-(\mathrm{f})$ is $20 \mu \mathrm{m}$. g) The distributions of gap distances between adjacent layers of expanded 3 and $10 \mathrm{~mm}$ thick nanofiber scaffolds. h) The distributions of layer thicknesses of expanded 3 and $10 \mathrm{~mm}$ thick nanofiber scaffolds. Both the gap distance and layer thickness were quantified using the Image I software based on SEM images.

giant cells per implant (Figure 7). The unexpanded nanofiber scaffolds showed a marginal increase in the depth of cell penetration from around $49.9 \mu \mathrm{m}$ at week 1 and 2 to $80 \mu \mathrm{m}$ at week 4 and 8 after implantation (Figure 7a). The expanded $3 \mathrm{~mm}$ thick scaffolds showed a steady increase of penetration depth from $67.1 \mu \mathrm{m}$ at week 1 , to $128.7 \mu \mathrm{m}$ at week 2 , to $171.5 \mu \mathrm{m}$ at week 4 , and further to $314.3 \mu \mathrm{m}$ at week 8 (Figure 7a). Similarly, the depth of cell penetration within expanded $10 \mathrm{~mm}$ thick scaffolds increased from $90.2 \mu \mathrm{m}$ at week 1 , to $94.3 \mu \mathrm{m}$ at week 2, to $186.8 \mu \mathrm{m}$ at week 4 , and further to $441.1 \mu \mathrm{m}$ at week 8 (Figure 7a). The aligned expanded $3 \mathrm{~mm}$ thick scaffolds showed a comparable depth of penetration as 3 and $10 \mathrm{~mm}$ thick scaffolds at week 4 and 8 . No newly formed blood vessels were seen within unexpanded scaffolds at all tested time points (Figure 7b). Differently, expanded 3 and $10 \mathrm{~mm}$ thick scaffolds showed a dramatic increase in blood vessel density from zero at week 1 , to 11.0 and 13.4 vessels $\mathrm{mm}^{-2}$ at week 2 , to 16.9 and 30.3 vessels $\mathrm{mm}^{-2}$ at week 4 , and further to 22.7 and 31.0 vessels $\mathrm{mm}^{-2}$ at week 8 (Figure $7 \mathrm{~b}$ ). Aligned expanded
$3 \mathrm{~mm}$ thick scaffolds showed a similar level of blood vessel density as 3 and $10 \mathrm{~mm}$ thick scaffolds.

The unexpanded nanofiber scaffolds showed an increase in the thickness of collagen fibrous capsules from $549.7 \mu \mathrm{m}$ at week 1, to $673.2 \mu \mathrm{m}$ at week 2, to $668.0 \mu \mathrm{m}$ at week 4 , and further to $840.6 \mu \mathrm{m}$ at week 8 (Figure 7c). In contrast, the thicknesses of capsules for expanded $3 \mathrm{~mm}$ thick scaffolds decreased from $523.7 \mu \mathrm{m}$ at week 1 , to $437.0 \mu \mathrm{m}$ at week 2, to $290.0 \mu \mathrm{m}$ at week 4 , and further to $348.9 \mu \mathrm{m}$ at week 8 (Figure 7c). The thickness of fibrous capsules of expanded $10 \mathrm{~mm}$ thick scaffolds was comparable to that of $3 \mathrm{~mm}$ thick scaffolds (Figure 7c). For the aligned expanded $3 \mathrm{~mm}$ thick scaffolds, the thickness of collagen fibrous capsules showed a slight increase from $128.9 \mu \mathrm{m}$ at week 1 to $254.2 \mu \mathrm{m}$ at week 2 , then remained almost constant at week 4 and week 8 (Figure 7c).

The number of giant cells per implant for unexpanded nanofiber scaffolds was about 16 at week 1,9 at week 2, 3 at week 4, and 6 at week 8 (Figure 7d). In comparison, the number of giant cells per implant for expanded $3 \mathrm{~mm}$ thick scaffolds 


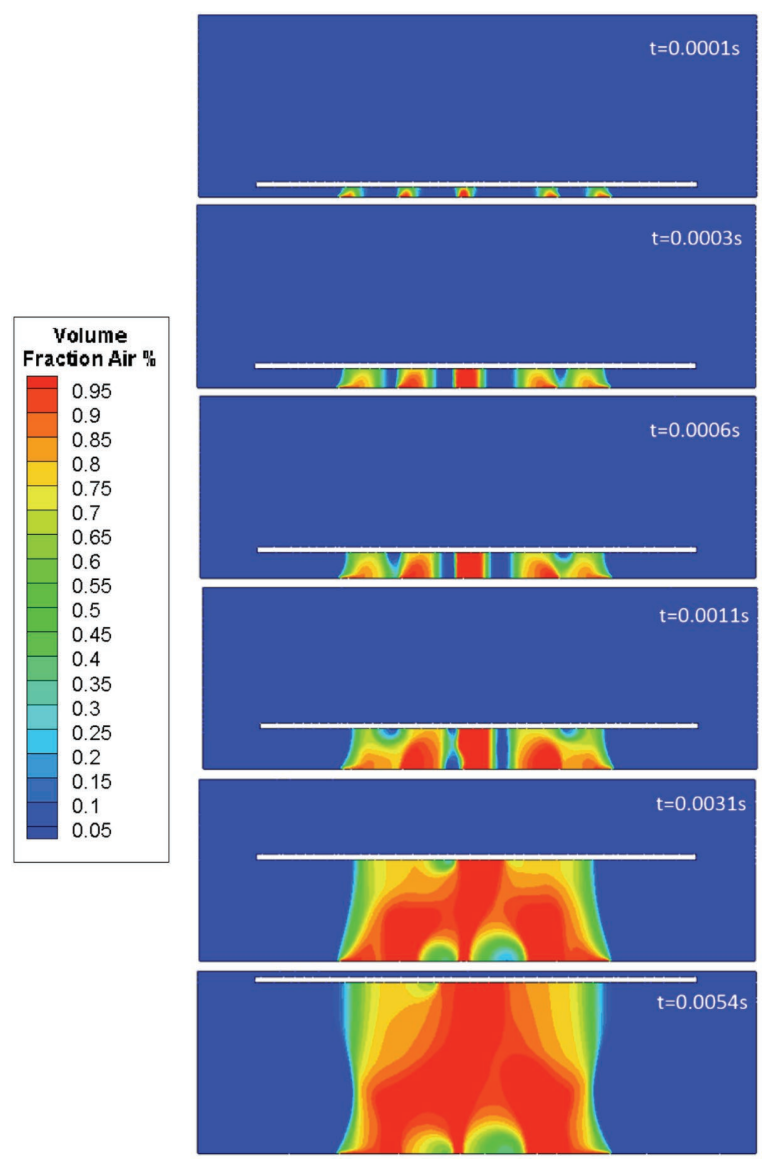

Figure 3. The simulated expanding process of electrospun nanofiber membranes.

was 16 at week 1, 28 at week 2, 31 at week 4, and 50 at week 8 (Figure 7d). Expanded $10 \mathrm{~mm}$ thick scaffolds showed a comparable number of giant cells per implant as $3 \mathrm{~mm}$ thick scaffolds at week 4 and 8 (Figure 7d). Differently, the number of giant cells per implant for aligned $3 \mathrm{~mm}$ thick scaffolds increased from week 1 (24) to week 2 (44) and then decreased to 20 at week 4 , and further to 13 at week 8 (Figure $7 d$ ).

\subsection{Immunostaining of Macrophage Phenotype}

In order to understand the phenotypes of infiltrated macrophages in scaffolds or in the surrounding areas, we performed immunostaining of scaffolds and surrounding tissues after explantation using three macrophage markers against CCR 7 (a surface marker for M1 macrophage phenotype), CD163 (a surface marker for M2 macrophage phenotype), and CD68 (a general macrophage marker). ${ }^{[14,15]}$ Figures S4-S7 (Supporting Information) show the spatiotemporal distribution of all macrophages, M1 macrophages, and M2 macrophages within scaffolds and surrounding tissues. As expected, unexpanded scaffolds displayed limited macrophage infiltration (Figure S4, Supporting Information), mostly appearing at week 4 and 8 . In the expanded scaffolds, macrophages penetration into scaffolds was apparent at week 1 , and increased thereafter.
The $10 \mathrm{~mm}$ thick scaffolds showed the greatest number of penetrating macrophages at week 8 (Figure S4, Supporting Information), with a similar spatiotemporal distribution of M1 and M2 phenotypes (Figures S5-S7, Supporting Information).

\subsection{Quantification of Macrophage Phenotype}

In order to better understand the phenotypes of infiltrated macrophages, we quantified the number of macrophages in each phenotype per immunohistochemical image $(40 \times)$ (Figure $8 \mathrm{a}-\mathrm{c}$ ). The unexpanded nanofiber scaffolds showed a slight increase in the number of $\mathrm{CD}^{+} 8^{+}$cells from 30 at week 1, to 33 at week 2, to 38 at week 4, and decreased to 24 at week 8 (Figure $8 \mathrm{a}$ ). Expanded $3 \mathrm{~mm}$ thick scaffolds showed a constant number (54) of $\mathrm{CD}^{+} 8^{+}$cells during the testing period. In contrast, $10 \mathrm{~mm}$ and aligned $3 \mathrm{~mm}$ thick scaffolds showed a dramatic increase in the number of $\mathrm{CD} 68^{+}$cells from 49 and 45 at week 1, to 56 and 46 at week 2, to 85 and 78 at week 4, and further to 88 and 91 at week 8 (Figure $8 \mathrm{a}$ ).

The number of $\mathrm{CCR}^{+}$cells for unexpanded nanofiber scaffolds was 40 at week 1, 41 at week 2, 42 at week 4, and 15 at week 8 (Figure $8 \mathrm{~b}$ ). Expanded $3 \mathrm{~mm}$ thick scaffolds showed a slight increase in the number of $\mathrm{CCR}^{+}$cells from 39 at week 1 , to 48 at week 2, then decreased to 34 at week 4 , and further to 25 at week 8 . In contrast, $10 \mathrm{~mm}$ and aligned $3 \mathrm{~mm}$ thick scaffolds showed a gradual increase in the number of CCR7 ${ }^{+}$cells from 31 and 32 at week 1, to 43 and 39 at week 2, to 47 and 58 at week 4, and further to 57 and 61 at week 8 (Figure 8b).

The numbers of $\mathrm{CD}_{163^{+}}$cells per snap shot for unexpanded nanofiber scaffolds were 27, 26, 30, and 20 at week 1, 2, 4, and 8 (Figure 8c). The numbers of $\mathrm{CD}_{163^{+}}$cells for expanded $3 \mathrm{~mm}$ scaffolds were similar at different time points $(41,49,46$, and $49 \mathrm{CD}_{163}{ }^{+}$cells per snap shot at week 1, 2, 4, and 8). Expanded $10 \mathrm{~mm}$ scaffolds showed an increase in the number of CD163 $3^{+}$ cells per snap shot from 46 at week 1 , to 47 at week 2, to 62 at week 4 , and further to 72 at week 8 (Figure $8 \mathrm{c}$ ). The corresponding numbers of $\mathrm{CD} 163^{+}$cells for the aligned $3 \mathrm{~mm}$ thick scaffolds were 35, 32, 48, and 57 at week 1, 2, 4, and 8. Figure 8d shows that expanded $10 \mathrm{~mm}$ thick scaffolds had higher ratios of M2/M1 at week 1 and 4 compared to unexpanded scaffolds. However, no significant difference in the ratio of M2/M1 was observed between the tested groups at week 8. In addition, expanded $3 \mathrm{~mm}$ thick scaffolds showed higher M2/M1 ratio than that of unexpanded counterparts.

\subsection{Heterogeneity of Multinucleated Giant Cells}

To understand the heterogeneity of multinucleated giant cells, we further analyzed the corresponding high-magnification images of Figures S4-S6 (Supporting Information). For random nanofiber scaffolds including unexpanded and expanded, the formed multinucleated giant cells expressed CD 68, CCR 7, and CD 163 markers (Figures S8-S10, Supporting Information). The expanded, aligned nanofiber scaffolds showed similar results (Figure S11, Supporting Information). We also quantified the number of CD $68^{+}, \mathrm{CCR} 7^{+}, \mathrm{CD} 163^{+}$, and the ratio of $\mathrm{CD} 163^{+} / \mathrm{CCR}^{+}$multinucleated giant cells (Figure S12, 

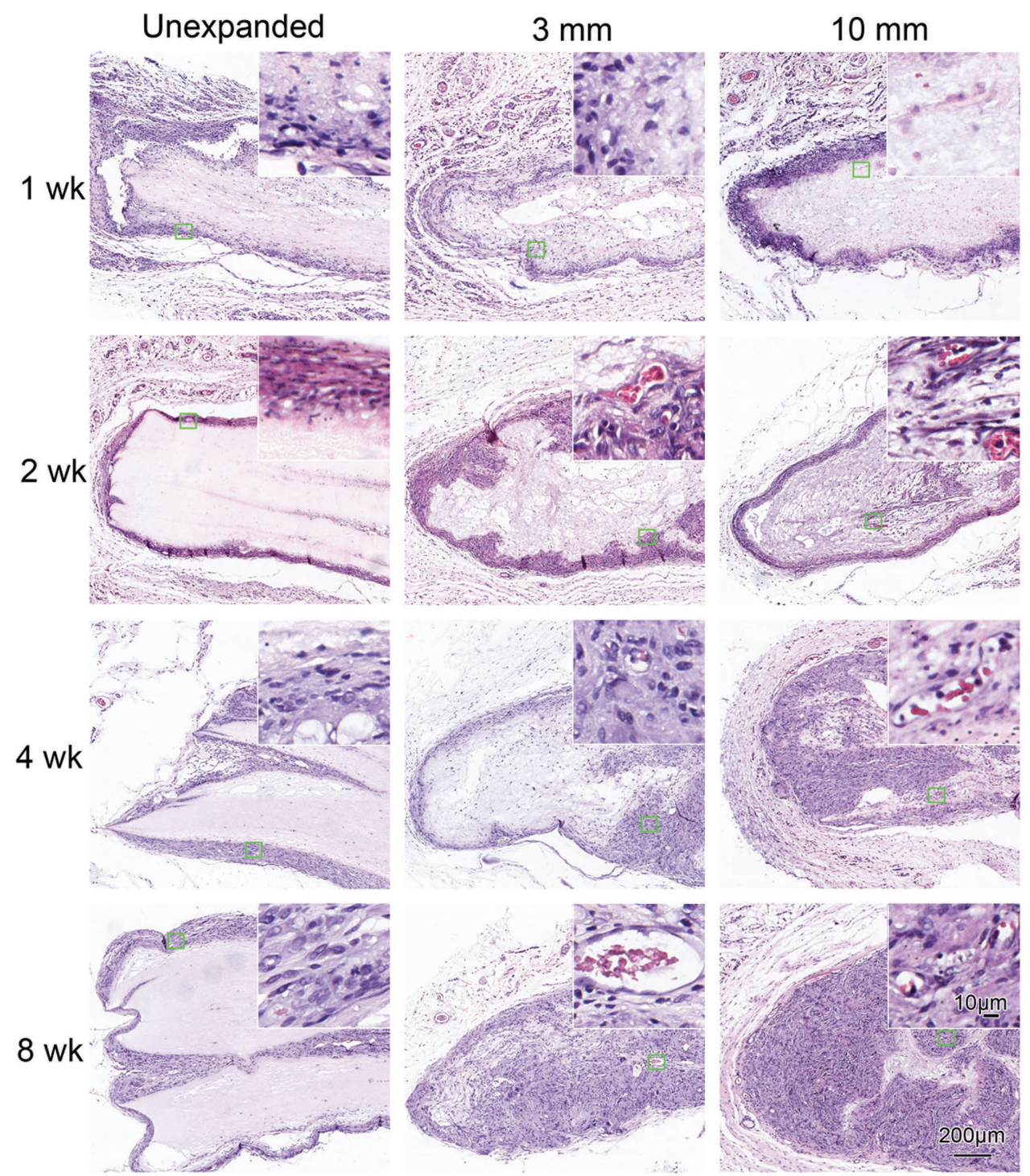

Figure 4. H\&E staining of random PCL nanofiber scaffolds and surrounding tissues. The unexpanded (raw) and expanded 3 and 10 mm thick nanofiber scaffolds were subcutaneously implanted to rats for 1, 2, 4, and 8 weeks. Insets show high magnification of green square areas in the corresponding images.

Supporting Information). Multinucleated giant cells expressed heterogeneous markers including CD 68, CCR7, and CD 163 (Figure S12a-c, Supporting Information). The number of $\mathrm{CD} 163^{+}$giant cells was usually higher than that of $\mathrm{CCR} 7^{+}$giant cells except for expanded $10 \mathrm{~mm}$ thick random scaffolds at week 2 and unexpanded scaffolds at week 4 (Figure S12d, Supporting Information). Expanded random nanofiber scaffolds with $3 \mathrm{~mm}$ thick showed the highest ratios of $\mathrm{CD} 163^{+} / \mathrm{CCR}^{+}$ multinucleated giant cells from week 2 to week 8 among the tested groups (Figure S12d, Supporting Information).

\subsection{Immunostaining of Inflammatory and Anti-Inflammatory Cytokines}

To further understand the effect of cytokines produced within scaffolds or in surrounding areas on the tissue regeneration, we performed immunostaining of scaffolds and surrounding tissues after explantation at week 1, 2, 4, and 8 using three different markers against IL-4 and IL-10 (anti-inflammatory cytokines) and TNF- $\alpha$ (pro-inflammatory cytokine). ${ }^{[16,17]}$ Figures S13-S16 (Supporting Information) show the spatiotemporal distribution of IL-4, IL-10, and TNF- $\alpha$ within scaffolds and surrounding areas. As expected, unexpanded scaffolds only displayed limited infiltration for IL-4 (Figures S13 and S16, Supporting Information), IL-10 (Figures S14 and S16, Supporting Information), and TNF- $\alpha$ (Figures S15 and S16, Supporting Information), and their secretion level remained unchanged from week 1 to week 8 . The IL-4 positive staining mainly located at the surrounding area of unexpanded scaffolds. For expanded scaffolds, IL-4 cytokines started expressing within the scaffolds from week 1 (Figure S13, Supporting Information). More IL-4 cytokines were accumulated within expanded scaffolds with increasing the implantation time (Figure S13, 

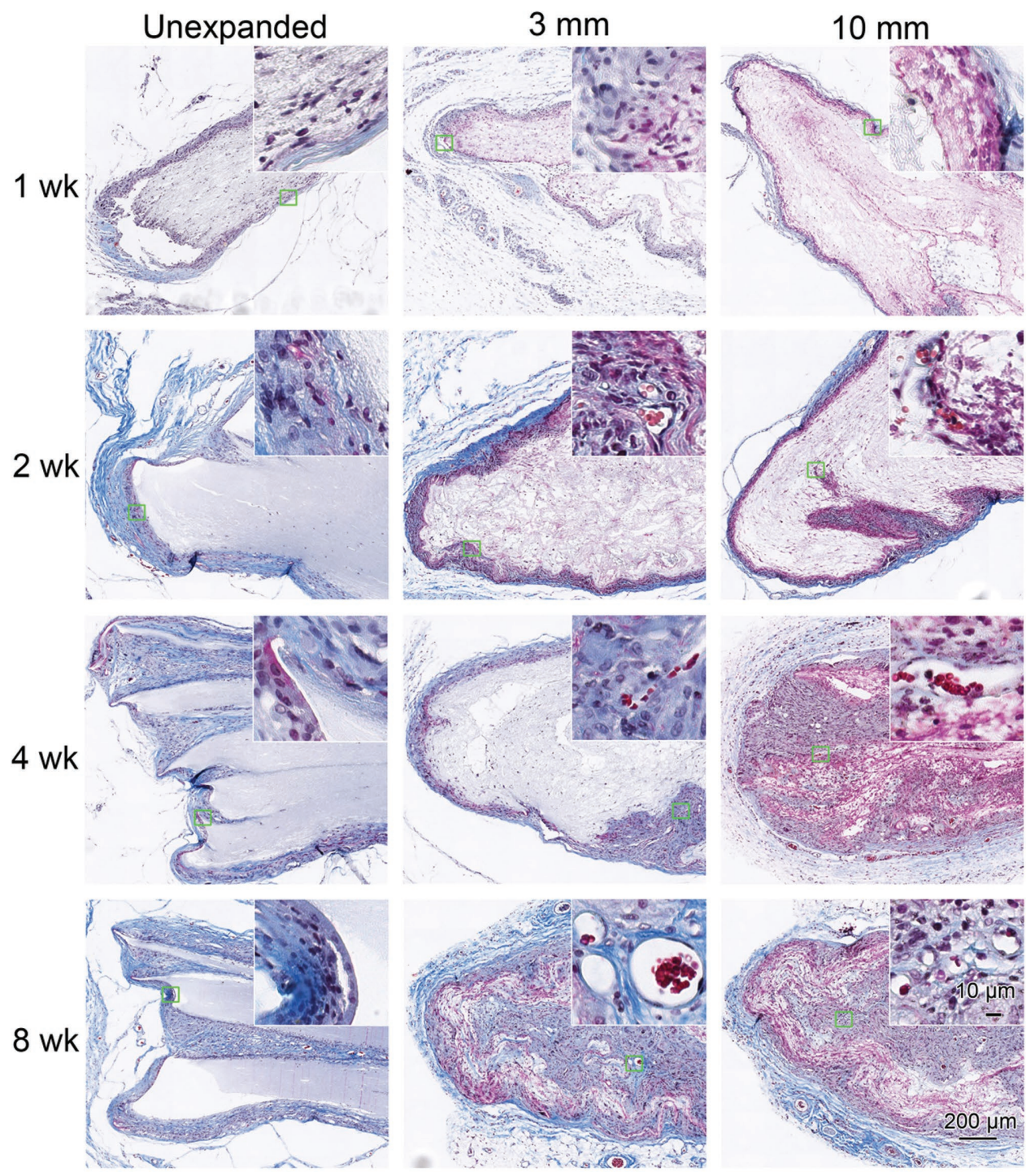

Figure 5. Masson's trichrome staining of random PCL nanofiber scaffolds and surrounding tissues. Masson's trichrome staining indicates collagen in blue, nuclei in black, and cytoplasm and keratin in red. The unexpanded (raw) and expanded 3 and $10 \mathrm{~mm}$ thick nanofiber scaffolds were subcutaneously implanted to rats for 1, 2, 4, and 8 weeks. Insets show high magnification of green square areas in the corresponding images.

Supporting Information). Obviously, the $10 \mathrm{~mm}$ thick scaffolds showed the highest level of IL-4 secretion at week 8 (Figures S13 and S15, Supporting Information). Intriguingly, IL-10 and TNF- $\alpha$ positive staining showed a similar spatiotemporal distribution within scaffolds as IL-4 (Figures S14-S16, Supporting Information). However, the level for TNF- $\alpha$ staining seemed much lower than that of IL-4 and IL-10.

\section{Discussion}

For tissue repair/regeneration, scaffolds/implants may play an important role for homing cells from surrounding healthy tissues and form new tissues to repair the defected tissues. ${ }^{[18]}$ Therefore, for sucessful in situ tissue regeneration, promoting cell ingrowth is one of the prerequisites for an ideal 3D scaffold. Studies have shown that cell infiltration is mainly determined by the architecture or structure of scaffolds. ${ }^{[19]}$ This is, in particular, critical for synthetic scaffolds as cells often fail to immediately disintegrate the artificial extracellular matrices for migration. ${ }^{[19]}$ Hence, cell ingrowth greatly relies on the porosity and pore size of scaffolds, which is especially true for non-biodegradable or slowly biodegradable materials. In a recent study, Jang and co-workers reported the cell infiltration within layered PCL nanofiber scaffolds after subcutaneous implantation in nude mice for six weeks, which were produced by electrospinning highly conductive solutions with additives of ionic salts. ${ }^{[10]}$ Cellular infiltration was observed, however, only one time point (six weeks) was examined. In addition, the porosity and the distribution of gap distances between adjacent layers were not reported. ${ }^{[10]}$ The present study demonstrated the fabrication of expanded 3 and $10 \mathrm{~mm}$ nanofiber scaffolds with porosities of $\approx 90 \%$ and $\approx 100 \%$, respectively. The gaps between most adjacent layers ranged from several micrometers to $\approx 100 \mu \mathrm{m}$ 

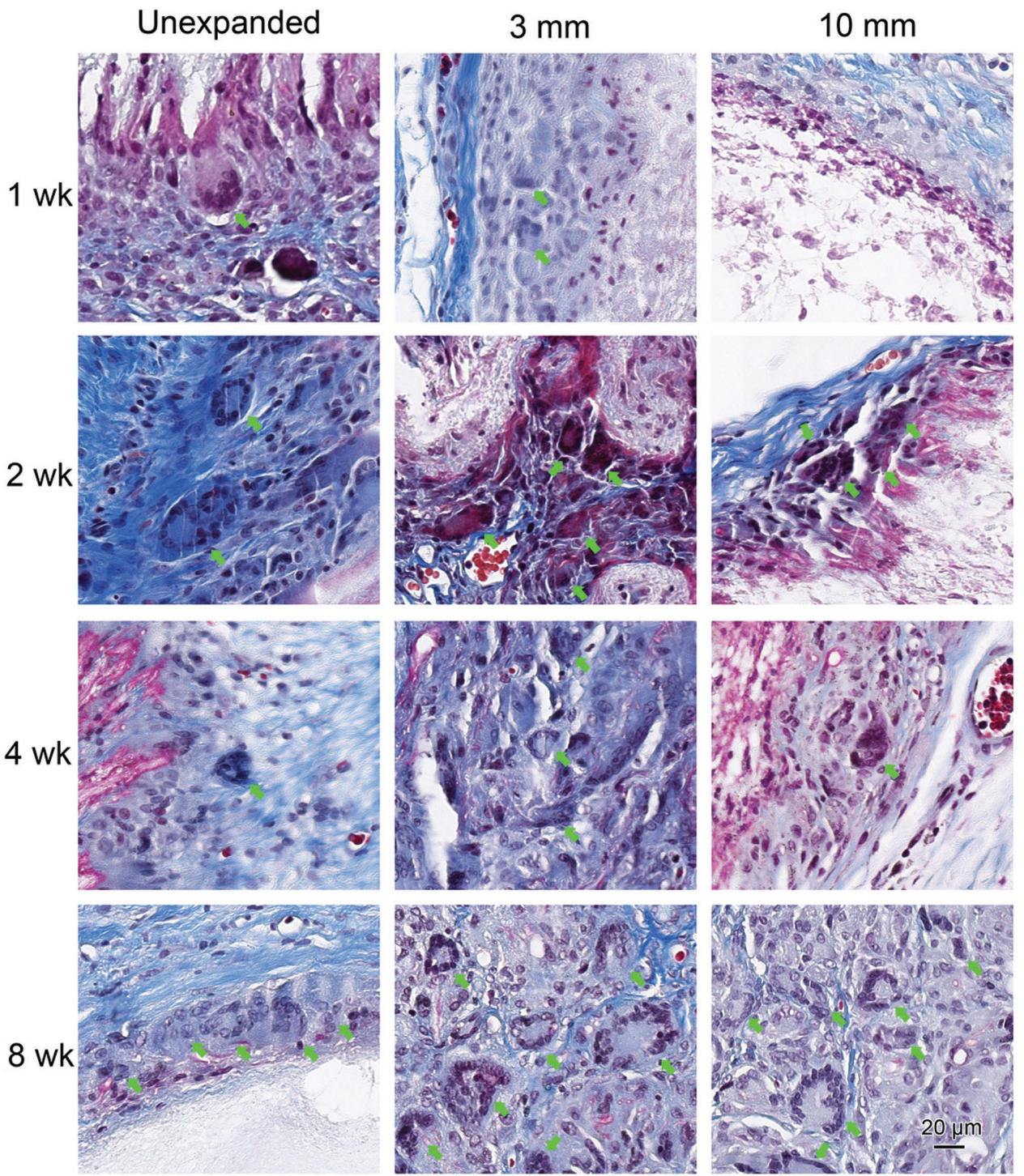

Figure 6. Multinucleated giant cells after random PCL nanofiber scaffold implantation. Masson's trichrome staining of random PCL nanofiber scaffolds after rat subcutaneous implantation. The rats were scarified at week 1, 2, 4, and 8 after surgery. Giant cells indicated by green arrows were found around and inside of PCL nanofiber scaffolds.

(Figure 2g). The dynamic process of cell penetration was shown from week 1 to week 8 , suggesting that cells may fully penetrate throughout the whole scaffold within eight weeks, mainly dependent on the porosity (Figures 4 and 5). Cells penetrated throughout the whole expanded 3 and $10 \mathrm{~mm}$ thick random scaffolds within eight weeks. The aligned expanded $3 \mathrm{~mm}$ thick scaffolds showed a similar penetration depth as expanded 3 and $10 \mathrm{~mm}$ thick random scaffolds at week 8. In addition, the unexpanded PCL nanofiber scaffolds exhibited very limited cellular infiltration on the surface layer, which agreed well with previous reports..$^{[13]}$

Sufficient oxygen and nutrient transport to the infiltrated cells within scaffolds is the key to form functional tissues in clinically relevant dimensions. ${ }^{[2]}$ Therefore, another important aspect for in situ tissue regeneration is neovascularization. Many attempts have been made to promote vascularization including scaffold design, endothelial cell pre-seeding, and incorporation of bioactive molecules. ${ }^{[21]}$ Among them, the pore architecture and porosity of scaffolds play a critical role on the formation of new blood vessles via sprouting from host vasculature. ${ }^{[22]}$ Beier and co-workers examined the actual pattern of vascularization in $\mathrm{PCL} /$ collagen nanofiber scaffolds using micro-CT scans and found the aligned scaffold showed a significantly smaller number of sprouting vessels but vascularization in the center of the constructs occurred considerably earlier than in the nonwoven scaffold. ${ }^{[23]}$ In a separate study, Andreopoulos and co-workers showed that aligned electrospun gelatin nanofibers containning bFGF had the highest vessel density (33 vessels $\mathrm{mm}^{-2}$ ) comparing to other groups including aligned fibers and random fibers with and without containing bFGF $\left(5\right.$ vessles $\mathrm{mm}^{-2}$ ) after implantation in the mouse's hindlimb for $21 \mathrm{~d} .{ }^{[24]}$ Park and co-workers also examined the vascularization of 1-1.5 mm thick ten-layered PCL/collagen nanofiber scaffolds formed by manually folding after subcutaneous 

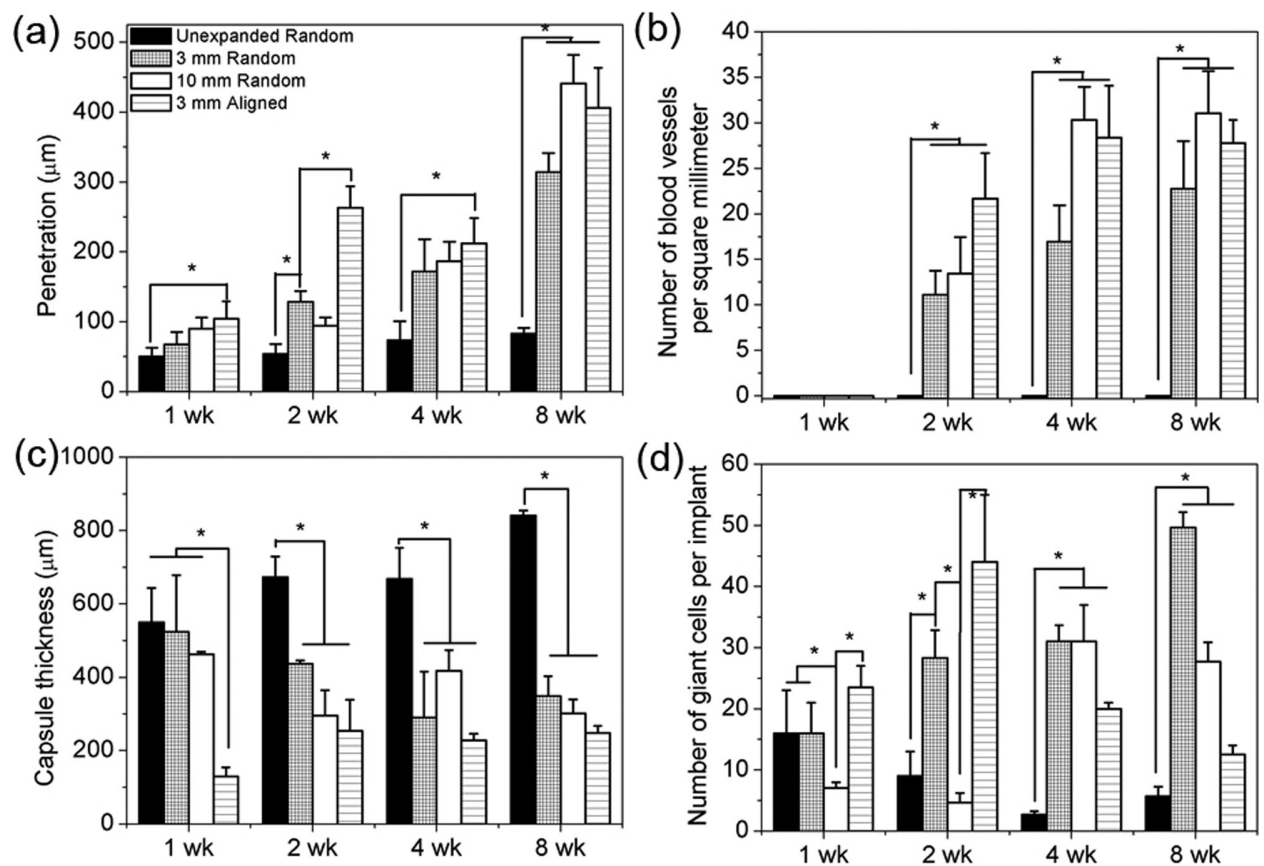

Figure 7. Quantification of histological analysis of PCL nanofiber scafflods after subcutaneous implantation. a) Cell penetration, b) blood vessels density, c) capsule thickness, and d) number of giant cells. The values were obtained by measuring six scanning images at 40× (objective lense) magnification for each specimen.

implantation in rats. ${ }^{[25]}$ Dunn and co-workers developed a laser cutting pores in traditional nanofiber membranes for enhancement of vascular ingrowth. ${ }^{[26]}$ In this work, no blood vessels were observed within unexpanded nanofiber scaffolds at all time points because of very limited cellular infiltration on the surface layer. Within expanded PCL nanofiber scaffolds

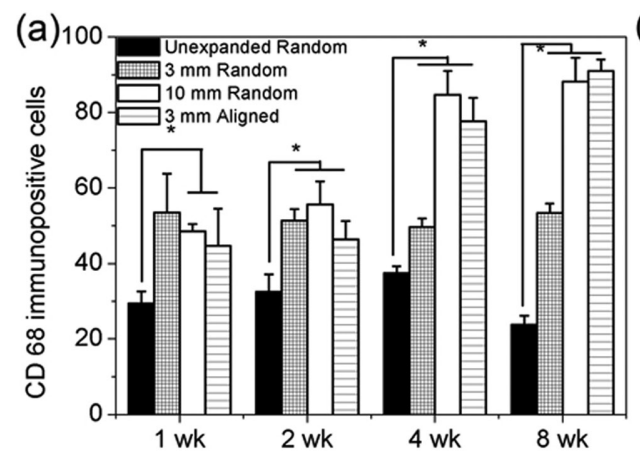

(b)

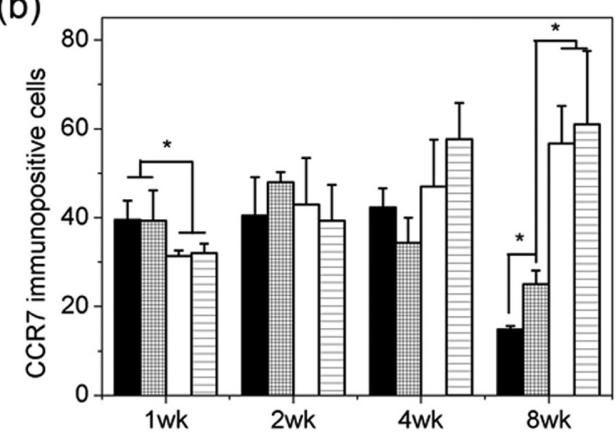

(c)

(d)
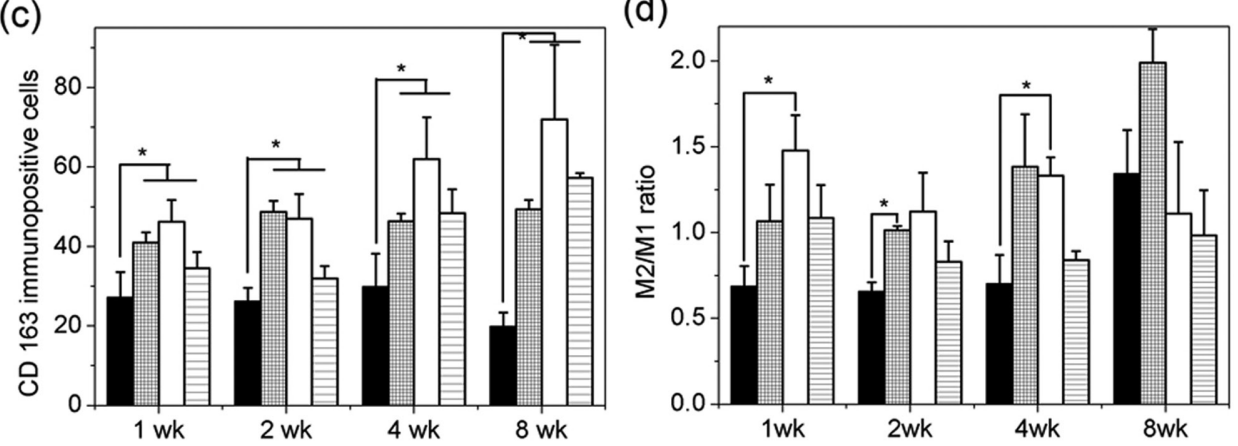

Figure 8. Quantification of immunhistological analysis of PCL nanofiber scafflods after subcutaneous implantation. a) CD 68, b) CCR 7 (M1), c) CD 163 (M2) immunpositve cells, and d) ratio of number of CD163 positive cells (M2)/number of CCR7 positive cells (M1). The values were obtained by measuring six scanning images at 40x (objective lense) magnification for each specimen. 
new blood vessels appeared at week 2 and blood vessel density increased with increasing implantation time and reached 30 vessels $\mathrm{mm}^{-2}$ at week 8 , which is comparable to the aligned gelatin nanofibers containing bFGF ( 33 vessels $\mathrm{mm}^{-2}$ ). In addition, expanded $10 \mathrm{~mm}$ thick scaffolds usually showed higher number of blood vessels per unit area within scaffolds than that of $3 \mathrm{~mm}$ thick ones (Figure 7b). The blood vessel density within aligned expanded $3 \mathrm{~mm}$ thick scaffolds was similar to that of $10 \mathrm{~mm}$ thick scaffolds at week 4 and 8 .

Implants require tissue engraftment and vascularization to generate functional tissues for replacement/repair/regeneration. Host response to implantated scaffolds is very important to determine their success after implantation. The biological response has been examined on various biomaterials with different sizes, compositions, and shapes. ${ }^{[27]}$ However, the host respone to electrospun nanofiber scaffolds was not fully illustrated and most of work was limited to the investigation of traditional 2D electrospun nanofiber membranes. Chew et al. examined host response of PCL nanofiber membranes and found that aligned nanofibers can minimize host response, enhance tissue-scaffold integration, and elicit a thinner fibrous capsule compared with random nanofibers. ${ }^{[28]}$ In the present study, expanded nanofiber scaffolds displayed thinner fibrous capsules compared to 2D nanofiber membranes. Expanded 3 and $10 \mathrm{~mm}$ thick scaffolds presented the similar thickness of fibrous capsules. The aligned expanded $3 \mathrm{~mm}$ thick scaffolds showed thinner fibrous capsules than expanded 3 and $10 \mathrm{~mm}$ thick scaffolds. Expanded nanofiber scaffolds showed the infiltration of macrophages in different phenotypes and induction of heterogenous multinucleated giant cells within the scaffolds, which could be critical for the neovascularization and regeneration process. ${ }^{[29-33]}$ The expanded $10 \mathrm{~mm}$ thick scaffolds had the highest number of infiltrating macrophages at week 8 (Figure S4, Supporting Information). The number of giant cells per implant was comparable for expanded 3 and $10 \mathrm{~mm}$ thick scaffolds at week 4 and 8 . Interestingly, expanded $3 \mathrm{~mm}$ thick scaffolds showed the highest M2/M1 ratio of multinucleated giant cells. In addition, the number of giant cells per implant for aligned $3 \mathrm{~mm}$ thick scaffolds was much lower compared to random 3 and $10 \mathrm{~mm}$ thick scaffolds at week 8 . The multinucleated giant cells present within aligned $3 \mathrm{~mm}$ thick scaffolds showed heterogeneous markers including CD 68, CCR7, and CD 163 similar to other scaffolds. Recent studies suggested that a porous polymer with interconnected pores $(\approx 40 \mu \mathrm{m}$ in size $)$ can form vascularized tissues with little or no fibrosis and good resoration of vascularity, whereas the same polymer in solid form triggers the classic foreign body reaction characterized by a dense, collagen fibrous capsule and low vascularity. ${ }^{[4]}$ Our results are in line with this finding as the expanded scaffolds show the distances of gaps between layers ranging from several micrometers to about $100 \mu \mathrm{m}$. Importantly, expanded nanofiber scaffolds showed IL-4 cytokine expression at week 1 and an increased IL-4 production within scaffolds with increasing the implantation time. Expanded $10 \mathrm{~mm}$ thick scaffolds demonstrated the highest expression of IL-4 within scaffolds at week 8 among all the tested scaffolds. IL-4 could be produced by $T$ helper 2 cells and guided the polarization of infiltrated macrophages, providing a pro-regenerative microenvironment. ${ }^{[35]}$ In addition, the incorporation of anti-inflammatory drugs to the nanofibers could further reduce the thickness of collagen fibrous capsules. ${ }^{[36]}$ The incorporation of immunmodulating agents to scaffolds for sustained release could temperoally control the phenotypes of macrophages infiltrated. ${ }^{[37]}$

\section{Conclusion}

We have developed a robust method for producing expanded nanofiber scaffolds with controlled size and thickness using a custermized mold during the modified gas-foaming process. We demonstrated the evident cellular infiltration and new blood vessel formation within expanded nanofiber scaffolds after subcutaenous implantation in rats. We also observed that expanded scaffolds elicited a thinner collagen fibrous capsule compared to unexpanded nanofiber scaffolds and promoted a regenerative response. Gap distances between adjacent layers, layer thickness, porosity, and fiber alignment mainly determined cellular infiltration, vascularization, and host response. In summary, such expanded nanofiber scaffolds hold great potential for use in tissue repair/regeneration in situ, the development of various 3D tissue models/constructs in vitro, and wound dressings.

\section{Experimental Section}

See the Supporting Information.

\section{Supporting Information}

Supporting Information is available from the Wiley Online Library or from the author.

\section{Acknowledgements}

This work was supported partially from startup funds from University of Nebraska Medical Center (UNMC), National Institute of General Medical Science (NIGMS) Grant No. 2P20 GM103480-06, and UNMC Regenerative Medicine Program Pilot Project Grant No. 37-1209-2004-007.

Received: July 26, 2016 Revised: September 6, 2016 Published online:

[1] J. H. Jang, O. Castano, H. W. Kim, Adv. Drug Delivery Rev. 2009, 61, 1065.

[2] S. G. Kumbar, S. P. Nukavarapu, R. James, L. S. Nair, C. T. Laurencin, Biomaterials 2008, 29, 4100.

[3] S. G. Kumbar, R. James, S. P. Nukavarapu, C. T. Laurencin, Biomed. Mater. 2008, 3, 034002.

[4] S. Ramakrishna, K. Fujihara, W. Teo, T. Yong, Z. Ma, R. Ramaseshan, Mater. Today 2006, 9, 40.

[5] G. Charras, E. Sahai, Nat. Rev. Mol. Cell Biol. 2014, 15, 813.

[6] E. L. S. Fong, S. Lambamedi-Cherradi, E. Burdett, V. Ramamoorthy, A. J. Lazar, F. K. Kasper, M. C. Farach-Carson, D. Vishwamitra, E. G. Demicco, B. A. Menegaz, H. M. Amin, A. G. Mikos, J. A. Ludwig, Proc. Natl. Acad. Sci. USA 2014, 110, 6500. 
[7] T. M. Dinis, R. Elia, G. Vidal, Q. Dermigny, C. Denoeud, D. L. Kaplan, C. Egles, F. Marin, J. Mech. Behav. Biomed. 2015, 41, 43.

[8] B. A. Blakeney, A. Tambralli, J. M. Anderson, A. Andukun, D. J. Lim, D. R. Dean, H. W. Jun, Biomaterials 2011, 32, 1583.

[9] H. Xu, S. Cai, L. Xu, Y. Yang, Langmuir 2014, 30, 8461.

[10] G. Jin, M. Shin, S. Kim, H. Lee, J. Jang, Angew. Chem., Int. Ed. 2015, $54,7587$.

[11] J. B. Lee, S. I. Jeong, M. S. Bae, D. H. Yang, D. N. Heo, C. H. Kim, E. Alsberg, I. K. Kwon, Tissue Eng., Part A 2011, 17, 2695.

[12] S. I. Jeong, N. A. Burns, C. A. Bonino, I. K. Kwon, S. A. Khan, E. Alsberg, J. Mater. Chem. B 2014, 2, 8116.

[13] J. Jiang, M. A. Carlson, M. J. Tewsink, H. Wang, M. R. MacEwan, J. Xie, ACS Biomater. Sci. Eng. 2015, 1, 991.

[14] S. F. Badylak, J. E. Valentin, A. K. Ravindra, G. P. McCabe, A. M. Stewart-Akers, Tissue Eng., Part A 2008, 14, 1835.

[15] B. N. Brown, J. E. Valetin, A. M. Stewart-Akers, G. P. McCbe, S. F. Badylak, Biomaterials 2009, 30, 1482.

[16] S. M. Opal, V. A. Depalo, Chest 2000, 117, 1162.

[17] C. A. Dinarello, Chest 2000, 118, 503.

[18] P. Danilevicius, L. Georgiadi, C. J. Pateman, F. Claeyssens, M. Chatzinikolaidou, M. Farsari, Appl. Surf. Sci. 2015, 336, 2.

[19] F. J. O'Brien, Mater. Today 2011, 14, 88.

[20] M. Lovett, K. Lee, A. Edwards, D. L. Kaplan, Tissue Eng., Part B 2009, 15, 353.

[21] N. M. S. Bettahalli, J. Vicente, L. Moroni, G. A. Higuera, C. A. Blitterswijk, M. Wessling, D. F. Stamatialis, Acta Biomater. 2011, 7, 3312.

[22] L. R. Madden, D. J. Mortisen, E. M. Sussman, S. K. Dupras, J. A. Fugate, J. L. Cuy, K. D. Hauch, M. A. Laflamme, C. E. Murry, B. D. Ratner, Proc. Natl. Acad. Sci. USA 2010, 107, 15211.

[23] D. Klumpp, M. Rudisile, R. I. Kuhnle, A. Hess, F. F. Bitto, A. Arkudas, O. Bleiziffer, A. M. Boos, U. Kneser, R. E. Horch, J. P. Beier, J. Biomed. Mater. Res., Part A 2012, 100, 2302.

[24] R. B. Montero, R. I. Vazquesz-Padron, S. M. Pham, G. D'Ippolito, F. M. Andreopoulos, Open J. Regener. Med. 2014, 3, 1.
[25] D. A. Barker, D. T. Bowers, B. Hughley, E. W. Chance, K. J. Klembczyk, K. L. Brayman, S. S. Park, E. A. Botchwey, JAMA Otolaryngol. Head Neck Surg. 2013, 139, 914.

[26] V. S. Joshi, N. Y. Lei, C. M. Walthers, B. Wu, J. C. Y. Dunn, J. Surg. Res. 2013, 183, 18.

[27] O. Veiseh, J. C. Doloff, M. Ma, A. J. Vegas, H. H. Tam, A. R. Bader, J. Li, E. Langan, J. Wyckoff, W. S. Loo, S. Jhunjhunwala, A. Chiu, S. Siebert, K. Tang, J. Hollister-Lock, S. Aresta-Dasilva, M. Bochenek, J. Mendoza-Elias, Y. Wang, M. Qi, D. M. Lavin, M. Chen, N. Dholakia, R. Thakrar, I. Lacik, G. C. Weir, J. Oberholzer, D. L. Gainer, R. Langer, D. G. Anderson, Nat. Mater. 2015, 14, 643.

[28] H. Cao, K. Mchugh, S. Y. Chew, J. M. Anderson, J. Biomed. Mater. Res., Part A 2010, 93A, 1151.

[29] M. Barbeck, A. Motta, C. Miglizresi, R. Sader, C. J. Kirkpatrick, S. Ghanaati, J. Biomed. Mater. Res., Part A 2016, 104, 413.

[30] M. Barbeck, J. Lorenz, A. Kubesch, N. Bohm, P. Booms, J. Choukroun, R. Sader, C. J. Kirkpatrick, S. Ghanaati, J. Oral Implant. 2015, 41, e238.

[31] M. Barbeck, S. Udeabor, J. Lorenz, M. Schlee, M. G. Holthaus, N. Raetscho, J. Choukroun, R. Sader, C. J. Kirkpatrick, S. Ghanaati, J. Oral Implant. 2015, 41, e212.

[32] M. Bareck, S. E. Udeabor, J. Lorenz, A. Kubesch, J. Choukroun, R. A. Sader, C. J. Kirkpatrick, S. Ghanaati, Ann. Maxillofac. Surg. 2014, 4, 150.

[33] S. Ghanaati, M. Barbeck, C. Orth, I. Willershausen, B. W. Thimm, C. Hoffmann, A. Rasic, R. A. Sader, R. E. Unger, F. Peters, C. J. Kirkpatrick, Acta Biomater. 2010, 6, 4476

[34] B. D. Ratner, Regener. Biomater. 2016, 3, 107.

[35] K. Sadtler, K. Estrellas, B. W. Allen, M. T. Wolf, H. Fan, A. J. Tam, C. H. Patel, B. S. Luber, H. Wang, K. R. Wagner, J. D. Powell, F. Housseau, D. M. Pardoll, J. H. Elisseeff, Science 2016, 352, 366.

[36] N. M. Vacanti, H. Cheng, P. S. Hill, J. D. Guerreiro, T. T. Dang, M. Ma, S. Watson, N. S. Hwang, R. Langer, D. G. Anderson, Biomacromolecules 2012, 13, 3031.

[37] S. Minardi, B. Corradetti, F. Taraballi, J. H. Byun, F. Cabrera, X. Liu, M. Ferrari, B. K. Weiner, E. Tasciotti, Ann. Biomed. Eng. 2016, 44, 2008. 